Proceedings of the 2012 Winter Simulation Conference

C. Laroque, J. Himmelspach, R. Pasupathy, O. Rose, and A. M. Uhrmacher, eds.

\title{
ABMS OPTIMIZATION FOR EMERGENCY DEPARTMENTS
}

\author{
Eduardo Cabrera \\ Emilio Luque \\ CAOS, Edifici Q1 \\ Universitat Autònoma de Barcelona (UAB) \\ E-08193, Bellaterra, SPAIN
}

\author{
Manel Taboada \\ Tomàs Cerdà Computer Science School \\ Universitat Autònoma de Barcelona (UAB) \\ E-08174 Sant Cugat, SPAIN
}

\author{
Francisco Epelde \\ Ma Luisa Iglesias \\ Hospital of Sabadell \\ Corporació Sanitària Parc Taulí \\ Parc Taulí, 1 \\ E-08208 Sabadell, SPAIN
}

\begin{abstract}
This article presents an agent-based modeling and simulation to design a decision support system for healthcare emergency department (ED) to aid in setting up management guidelines to improve it. This ongoing research is being performed by the Research Group in Individual Oriented Modeling at the Universitat Autònoma de Barcelona with close collaboration of the hospital staff team of Sabadell. The objective of the proposed procedure is to optimize the performance of such complex and dynamic healthcare EDs, which are overcrowded. Exhaustive search optimization is used to find the optimal ED staff configuration, which includes doctors, triage nurses, and admission personnel, i.e., a multi-dimensional and multi-objective problem. An index is proposed to minimize patient stay time in the ED. The model is implemented using NetLogo. The results obtained by using alternatives Monte Carlo and Pipeline schemes are promising. The impact of these schemes to reduce the computational resources used is described.
\end{abstract}

\section{INTRODUCTION}

Health is one of the most appraised gifts for human beings; therefore, it is crucial to preserve it. Healthcare units were designed to take charge of it. Healthcare systems have evolved during the previous decades, specifically emergency departments (ED). ED is a primary healthcare department, usually the main entrance to the hospital, and a key component of the whole healthcare system. EDs are semi-autonomous units that are open and staffed 24 hours per day, 365 days per year, including holidays. The original mission of EDs is to primarily handle only emergent situations. However, ED visits include a wide range of illnesses and injuries, i.e., truly emergencies, urgent, semi-urgent, and non-urgent cases. EDs have increased their resources to attend all of those cases, becoming large, complex and dynamic units. In spite of such an increase, patients continue to suffer, since they do not have access to ad hoc healthcare, in some cases due to the inefficiencies of the EDs functioning. As a result of this, EDs are overcrowded and the length of stay (LoS) of patients has increased, whereas quality of service has decreased. Indeed, overcrowding of EDs is a worldwide issue, and a national crisis in the US (Institute of Medicine (IoM) and Committee on the Future of Emergency Care in the United States Health System 2007). Although ED overcrowding is not a new topic, since it was documented in the literature 20 years ago (Lynn and Kellermann 1991), 


\section{Cabrera, Luque, Taboada, Epelde, and Iglesias}

there is not a solution to this long and growing issue yet. Despite that EDs are under this overcrowding phenomena, they have suffered budget reductions. Therefore, new techniques and paradigms should be found in order to deal with such overcrowded condition. ED managers require different and fresh solutions, because society demands not only care, quality and service, but also the best care, quality and service. A direct solution to this issue is increasing the size of EDs. However, this straightforward solution is limited by the facility, number of staff (doctors, nurses, technicians) and services (computing, communication, radiology, laboratory), and it is not the best approach. Also, healthcare managers have to maximize the use of healthcare resources, whereas being constrained by limited budget, in order to minimize patient LoS, while increase satisfaction of the patients, i.e., to optimize the performance of the ED. The resource planning of an ED is complex activity, since it is not linear, and it varies depending on time, day of week and season. The ability to simulate special situations such as seasonal increases in ED demand can be useful for the efficient use of resources. There are no standard models to describe a complex system. Simulation becomes an important tool for modeling systems including many elements as well as interdependencies among the elements, and/or considerable variability. Discrete event simulation (DES), system dynamics (SD) and agent-based modeling and simulation (ABMS) are the three main approaches used to simulate healthcare systems. There are a large and growing body of literature describing the use of DES and SD models in ED studies, but the use of ABMS for this purpose is few; although healthcare systems are based on human actions and interactions that are quite difficult to model with DES, and can be more properly modeled with ABMS.

This article presents the results of an ongoing research project that is being carried out by the Research Group in Individual Oriented Modeling (IoM) in the Universitat Autònoma de Barcelona (UAB), with the participation of the ED managers of the Hospital of Sabadell (one of the most important Hospitals in Spain that gives care service to an influence area of 500,000 people, and attends 160,000 patients/year in the ED). The general objective of the project is to develop an ED simulator that, used as a decision support system (DSS), aids the managers of EDs in setting up strategies, and management guidelines to enhance the efficiency of such EDs. The specific objective is to implement in the proposed DSS model an algorithm to optimize the number of staff members of EDs, including admission staff, triage nurses, and doctors, under certain economical and operational constrains.

The Emergency Department model defined herewith is a pure Agent-Based Model (ABM), and so is formed entirely of the rules governing the behavior of the individual agents that populate the system. The system behavior emerges as a result of local level actions and interactions. This model describes the complex dynamics found in an ED, representing each individual and system as an individual agent.

The rest of this article is organized as follows; Section 2 describes the literature review. The ED model is succinctly described in Section 3, the mathematical-computational model of the ED are presented in Section 4, the optimization problem of interest, the experiment, and the results of three simulation optimization techniques are given in Section 5, the proposed methodology to find the optimum is described in Section 6. Finally, the conclusions and future work are presented in Section 7.

\section{LITERATURE REVIEW}

In 1979 computer simulation was applied to hospital systems to improve the scheduling of staff members (Hancock and Walter 1979), and in Saunders, Makens, and Leblanc (1989) the aim was to quantify the impact that the amount of staff members and beds had on patient throughput time. Moreover, a survey of discrete-event simulation in healthcare clinics was presented in 1999 Jun, Jacobson, and Swisher (1999).

Although, discrete-event simulation is widely used in simulating healthcare systems, agent technology is most suited to be used in healthcare applications, because it characterizes better the operation of complex systems such as EDs. There is a relevant article which uses ABM to simulate the work flow in ED (Wang 2009). It focus on triage and radiology processes, but not real data is used, the acuity of patients are not considered, and healthcare providers do not always serve patients in a first-come-first-serve basis. Simulation optimization is used to improve the operation of ED in Ruohonen, Neittaanmaki, and Teittinen 


\section{Cabrera, Luque, Taboada, Epelde, and Iglesias}

(2006), using a commercial simulation package, and Ahmed and Alkhamis (2009) combines simulation with optimization, which involves a complex stochastic objective function under deterministic and stochastic sets of restrictions. Also, in Weng, Cheng, Kwong, Wang, and Chang (2011) system simulation is used to improve the flow of the ED using an index to measure the degree of congestion of ED. Previous works on modeling healthcare systems have focused on patient scheduling under variable pathways and stochastic process durations, the selection of an optimal mix for patient admission in order to optimize resource usage and patient throughput (Hutzschenreuter et al. 2008). Work has been performed evaluating patient LoS under the effects of different ED physician staffing schedules, and the only one found until now that utilizes real data is Jones and Evans (2008) or patient diversion strategies (Laskowski and Mukhi 2008). An evolutionary multi-objective optimization approach is using for dynamic allocation of resources in hospital practice (Hutzschenreuter, Bosman, and Poutré 2009), while in Persson, Davidsson, Johansson, and Wernstedt (2005) it was found that combining agent-based approaches and classical optimization techniques complement each other.

This proposal addresses many of the issues surrounding the modeling and simulation of a healthcare emergency department using agent-based paradigm, where the efficiency of agents in this area has not been totally explored yet. The basic rules governing the actions of the individual agents are defined in an attempt to understand micro level behavior. The macro level behavior, that of the system as a whole, emerges as a result of the actions of these basic building blocks, from which an understanding of the reasons for system level behavior can be derived (Stainsby, Taboada, and Luque 2009).

\section{AGENT-BASED MODEL OF EMERGENCY DEPARTMENT}

In this Section, a brief description of the proposed Agent-Based Model (ABM) is presented. The Emergency Department model defined by this work is a pure ABM, thus it is formed entirely of the rules governing the behavior of the individual agents which populate the system, no higher level behavior is modeled. The system behavior emerges as a result of local level actions and interactions. This model describes the complex dynamics found in an ED, representing each individual and system as an individual agent. Two distinct kinds of agents have been identified, active and passive. Active agents represent the individuals involved in the ED, in this case all human actors, such as patients and ED staff (admission staff, nurses, doctors, etc). Passive agents represent services and other reactive systems, such as the information technology (IT) infrastructure or services used for performing tests. Moore State machines are used to represent the actions of each agent. This takes into consideration all the variables that are required to represent the many different states that such individual (a patient, a member of hospital staff, or any other role) may be in throughout the course of their time in a hospital emergency department. The change in these variables, invoked by an input from an external source, is modeled as a transition between states. In some specific cases the state machine involves probabilistic transitions, where a given combination of current state and input has more than one possible next state. Which transition is made is chosen at random at the time of the transition, weights on each transition provide a means for specifying transitions that are more or less likely for a given individual. Probabilities may be different for each agent. In this way heterogeneity is provided to agents as people, since agent behavior can be probabilistically defined external to their state. The communication between individuals is modeled as the inputs that agents receive and the outputs they produce, both implicitly and explicitly. The communication model represents three basic types of communication. The first type is 1-to-1 communication, such as between two individuals, for instance admission staff and patient, where a message has a single source and a single destination, as well as between patients, or staff personnel. The second type is 1-to-n communication, where a message has a single source and a specific set of recipients, for example when a doctor communicates with both patient and his companion, or when doctor communicates with other doctors and nurses. The last type is 1-to-location communication, where a message has a single source, but it is received by every agent within a certain area or location. This occurs when a triage nurse sends a message to the patients in the waiting room, through the loudspeaker system. In order to control the 
agent interaction, the physical environment in which these agents interact also has to be modeled, such as admissions, triage box, the waiting room, and consultation suits. A detailed description of the emergency department model using the agent-based paradigm can be found in the following article (Taboada et al. 2011)

\section{MATHEMATICAL-COMPUTATIONAL MODEL OF EMERGENCY DEPARTMENT}

\subsection{Multi-Objective Optimization}

From the mathematical point of view, the optimization problem stated in Section 1 is to optimize the number of staff members of an ED under specific economical and operational constraints. This problem can be formulated as a multi-objective optimization problem which is formally defined as follows: Find the vector $\vec{x}^{*}=\left[x_{1}^{*}, x_{2}^{*}, \ldots, x_{n}^{*}\right]^{T}$ which optimizes the vector function:

$$
\begin{array}{ll}
\max / \min & \vec{f}(\vec{x})=\left[f_{1}(\vec{x}), f_{2}(\vec{x}), \ldots, f_{k}(\vec{x})\right]^{T} \\
\text { subject to } & g_{i}(\vec{x}) \leq 0 \quad i=1,2, \ldots, m \\
& h_{i}(\vec{x})=0 \quad i=1,2, \ldots, p
\end{array}
$$

where $f_{i}$ is the $i$-th objective function. $x_{i}^{*}$ is a discrete vector, global sampling of $\vec{x}_{i}^{*}$ is possible to get, but not all of $f_{i}\left(x_{i}^{*}\right)$ can be evaluated exactly, and must be estimated via a simulation procedure. Since several objective functions exist, instead of looking for a single solution, an acceptable or trade-off solutions it is trying to find them. It is say that a vector of decision variables $\vec{x}^{*} \in P O$ is Pareto optimal if $\nexists$ another $\vec{x} \in P O$ such that $\left.f_{i}(\vec{x}) \leq f_{i}\left(\vec{x}^{*}\right) \quad \forall i=1, \ldots, k\right)$ and $f_{j}(\vec{x})<f_{j}\left(\vec{x}^{*}\right)$ for at least one $j$. $P O$ represents the feasible region of the problem, i.e., where the constraints are satisfied. The goal of the optimization procedure is

to identify the staff members of the EDs that optimize their performance, which needs to be estimated via a simulation due to the complexity of EDs. Optimization via simulation is a difficult task (Fu 2002). As simulations are usually computationally expensive, even estimating $f_{i}\left(x_{i}^{*}\right)$ at a single point in Equation (1) may require substantial effort, which implies that only some scenarios would be explored. Three different alternatives used in this work are briefly described following.

\subsection{Exhaustive Search}

Exhaustive search (ES) is used for discrete and combinatorial problems in which no efficient solution technique is known. In order to determine the solution it might be necessary to test each possibility and verify if it satisfies the statement of the problem. If there exists a solution, then ES technique will always find such solution. Nevertheless, the computational cost is high and proportional to the number of possible solutions which could be quite large, i.e., a combinatorial explosion.

\subsection{Monte Carlo}

Monte Carlo (MC) is a statistical sampling method used to approximate solutions to quantitative problems. It is widely used when it is infeasible to compute an exact solution using a deterministic algorithm, there are many degrees of freedom, or incertitude in the input data. MC method does not suffer from the curse of dimensionality. In order to reduce the standard error, different techniques could be used, among them are increasing the sample size and variance reduction.

\subsection{Pipeline}

This technique is usually known as assembly line. It consists of several ordered stages. The output of one stage is the input of the next one. Once the second stage is working, the first stage is attending the next input, and so on. Therefore, when the hole pipeline is full, one output per time is obtained. All stages could be configured to have more than one unit, i.e., parallel stages. So, not only the throughput but also the 
pipeline of the system is enhanced. However, when the process between each stage cannot be synchronized the use of buffers ought to be necessary. The pipeline time can be expressed by Equation (2), where $S_{i}$ represents each stage of the pipeline.

$$
\text { Pipeline time }=\frac{1}{\sum \frac{1}{S_{1}}}+\frac{1}{\sum \frac{1}{S_{2}}}+\ldots+\frac{1}{\sum \frac{1}{S_{k}}}
$$

\section{OPTIMIZATION}

\subsection{The Problem}

The optimization problem considered in this paper and expressed by Equation (1) aims to find the best ED staff configuration: doctors (D), triage nurses (N), and admissions (A), which optimize the ED's functioning. The simplified patient flow in the ED is defined as follows: Patients arrive to the ED on their own, and waits to be attended in the admission area. Then, patients stay in the first Waiting Room (WR), WR1, until a triage nurse calls them. After the triage process, patients pass to a second WR2, and stay there until a free doctor calls them to begin the diagnosis and treatment phase, depending on the patient's symptoms and physical condition, as well as prescribed tests. At the end, patients are discharged from the ED. Even though realistic treatment is based on the acuity of patients they have the same path throughout the ED at this moment in this research project.

For simplicity, only four different types of active agents are considered: patients, admission staff, nurses, and doctors. Staff considered characteristics are shown in Figure 1 and Table 1. Taking into account: a) that each of the personnel staff has two levels of expertise: low and high, labeled as Junior (J) and Senior (S), respectively. Figure 1 and Table 1 show, as well, the combinatorial problem for admission personnel, nurses and doctors; b) that the Junior staff will require more time to finish their own duty than Seniors; c) Senior staff have higher salaries. The time, and salary of each four staff, and the number of them, from 1 up to 3 or 4 , are shown in Table 1 . Figures $1 \mathrm{a}, 1 \mathrm{~b}$, and 1c, and Table 1 synthesize the combinatorial or multi-dimensional problem of interest (where each variable or in this case ED staff member represents one dimension, three dimension up to now, plus the patients arrival, i.e., the input to the ED, shown in Figure 2). As mentioned above the objective is to identify the combination numbers of staff members of ED that optimize its performance. The expected value of the latter needs to be estimated via simulation due to the complexity of EDs (as discussed previously).

Table 1: Staff members with their associated salary and time according to their kind, and the maximum number of them.

\begin{tabular}{lccccc}
\hline & \multicolumn{2}{c}{ Salary $(€)$} & \multicolumn{2}{c}{ Time (ticks) } & Number \\
\hline & Senior $(\mathbf{S})$ & Junior $(\mathbf{J})$ & Senior $(\mathbf{S})$ & Junior $(\mathbf{J})$ & \\
\hline Doctor (D) & 1,000 & 500 & 260 & 350 & $1-4$ \\
\hline Nurse (N) & 500 & 350 & 90 & 130 & $1-3$ \\
\hline Admin (A) & 200 & 150 & 20 & 35 & $1-3$ \\
\hline
\end{tabular}

\subsection{Generation of Samples of ED's Staff Configuration}

The ED simulator for this work is implemented in the agent-based simulation environment NetLogo (Wilensky 1999). The simulator is used as a black box, even though the more realistic the simulator is, the better are the results and the optimizations. The present scenario adopted for the experiments is to simulate patients moving through a simplified ED that includes four primary areas: admissions, three triage boxes, two waiting rooms, and the four diagnosis and treatment boxes. For simplicity, only four different types of active agents are considered: patients, admission staff, nurses, and doctors. ED staff considered 


\begin{tabular}{ccc}
\hline $\mathbf{A D}_{1}$ & $\mathbf{A D}_{2}$ & $\mathbf{A D}_{3}$ \\
\hline $\mathrm{AS}$ & - & - \\
\hline $\mathrm{AJ}$ & - & - \\
\hline $\mathrm{AS}$ & $\mathrm{AS}$ & - \\
\hline $\mathrm{AJ}$ & $\mathrm{AJ}$ & - \\
\hline $\mathrm{AS}$ & $\mathrm{AJ}$ & - \\
\hline $\mathrm{AS}$ & $\mathrm{AS}$ & $\mathrm{AS}$ \\
\hline $\mathrm{AJ}$ & $\mathrm{AJ}$ & $\mathrm{AJ}$ \\
\hline $\mathrm{AS}$ & $\mathrm{AJ}$ & $\mathrm{AJ}$ \\
\hline $\mathrm{AS}$ & $\mathrm{AS}$ & $\mathrm{AJ}$ \\
\hline
\end{tabular}

(a) 9 Admission (A) personnel staff configurations. AD $i$ represents Admission Den $i$.

\begin{tabular}{ccc}
\hline $\mathbf{T R}_{1}$ & $\mathbf{T R}_{2}$ & $\mathbf{T R}_{3}$ \\
\hline NS & - & - \\
\hline NJ & - & - \\
\hline NS & NS & - \\
\hline NJ & NJ & - \\
\hline NS & NJ & - \\
\hline NS & NS & NS \\
\hline NJ & NJ & NJ \\
\hline NS & NJ & NJ \\
\hline NS & NS & NJ \\
\hline
\end{tabular}

(b) 9 Nurse $(\mathrm{N})$ configurations. TR $i$ represents Triage Room $i$.

\begin{tabular}{cccc}
\hline DR $_{1}$ & DR $_{2}$ & DR $_{3}$ & $\mathbf{D R}_{4}$ \\
\hline DS & - & - & - \\
\hline DJ & - & - & - \\
\hline DS & DS & - & - \\
\hline DJ & DJ & - & - \\
\hline DS & DJ & - & - \\
\hline DS & DS & DS & - \\
\hline DJ & DJ & DJ & - \\
\hline DS & DJ & DJ & - \\
\hline DS & DS & DJ & - \\
\hline DS & DS & DS & DS \\
\hline DJ & DJ & DJ & DJ \\
\hline DS & DJ & DJ & DJ \\
\hline DS & DS & DJ & DJ \\
\hline DS & DS & DS & DJ \\
\hline
\end{tabular}

(c) 14 Doctor (D) configurations. DRi represents Diagnosis Room $i$.

Figure 1: Combinations of sanitarian staff: Admission (A) personnel, Nurses (N) and Doctors (D). Two levels of expertise: Junior (J), and Senior (S).

characteristics are shown in the Figure 1, and the basic patient attributes set as well as the simple patient flow in the ED are described previously.

In this experiment an average input of 400 patients has been assumed, which is the real average number of incoming patients that the heads of ED of the Hospital of Sabadell (HS) have reported. Patients arrive at the ED after a certain time step, and with different probabilities from 20 and $80 \%$ (P, these probability values were used to resemble the randomness of the incoming patients to the EDs and set different scenarios). Even with this very reduced setting of an ED, the search space is large. For example, the search space has 4,536 (which results of assuming a combination of two level of expertise, Junior or Senior, of up to four doctors, three nurses, three admissions [shown in Figures 1c, 1b, 1a], and four different probabilities of patients arrival, i.e., $14 \times 9 \times 9 \times 4$ ) combinations to find out which is the best or optimum that minimize a desire index, under some restrictions. The period simulated was 24 hrs., one day, which represent 24,000 ticks for all the experiments, as well as the same random seed, and 400 patients. This real average number of incoming patients daily hourly is shown in Figure 2 as well as its pattern.

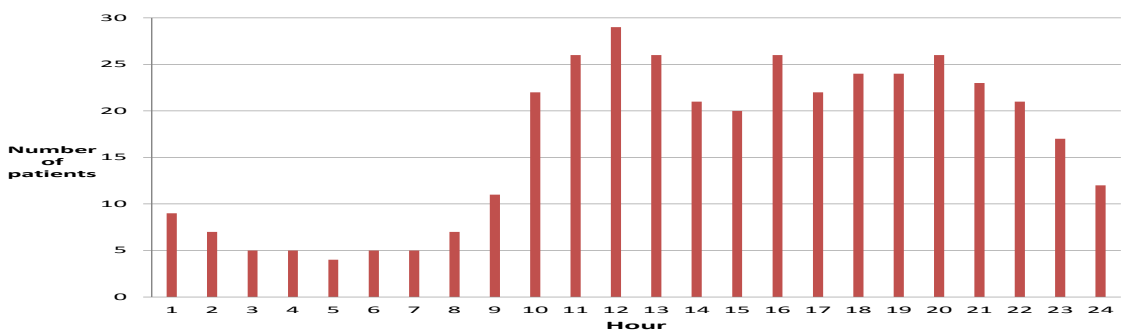

Figure 2: 400 is the real average number of incoming patients daily, hourly and its pattern.

In order to evaluate the utility of the proposed agent-based ED simulator for optimizing the resources of HS's ED, an index, defined as the minimum patient LoS in the latter, with a budget of 3,500€ per patient, was set. All simulations were done using the simulator already explained, in particular using serially the BehaviorSpace tool of NetLogo, on an IBM cluster, which has 32 compute nodes with $2 \times$ Dual-Core Intel(R) Xeon(R) CPU 5,160 at $3.00 \mathrm{GHz}$, with $12 \mathrm{~GB}$ of RAM, and 4MB of L2 share cache (2x2). 


\subsection{Experiment}

The mentioned index was used as a metric for the patient LoS in the ED, with budget constraint less or equal to $3,500 €$. This cost restriction consists of the salary of each sanitarian staff that constitutes the corresponding configuration and can be represented as follows: Cost $=\sum \beta_{i j} \times \lambda_{i j}$; where $\beta=$ person and $\lambda=$ salary, $i=D, N, A$ and $j=S, J$. The index is expressed mathematically in Equation (3).

$$
\begin{array}{ll}
\text { Minimize length of stay }(\mathrm{LoS}) & f(D, N, A) \\
\text { subject to } & C o s t \leq 3,500
\end{array}
$$

The first approach in searching for the optimum was done through an exhaustive search technique. Even though it implies a lot of search time, it is guaranteed that the optimum will be found, as stated in Section 4.2.

\subsection{Results and Discussion}

The results of the simulation are shown in Figure 3, where the blue rounded points are the staff configurations that satisfy the budget constraint, while the green and red asterisks are the average minimum LoS for each case. Only when patient arrival probability, P, 20\% and $80 \%$, staff configurations that led to the minimum $\mathrm{LoS}$ are presented in Figures $3 \mathrm{a}$ and $3 \mathrm{~b}$, as well as the associated cost of each staff configuration in Tables 2 and 3 , respectively.

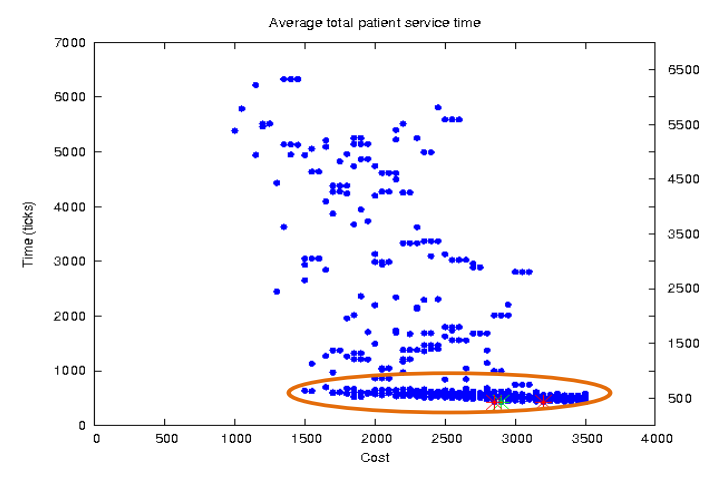

(a) Average minimum LoS with $\mathrm{P}=20 \%$.

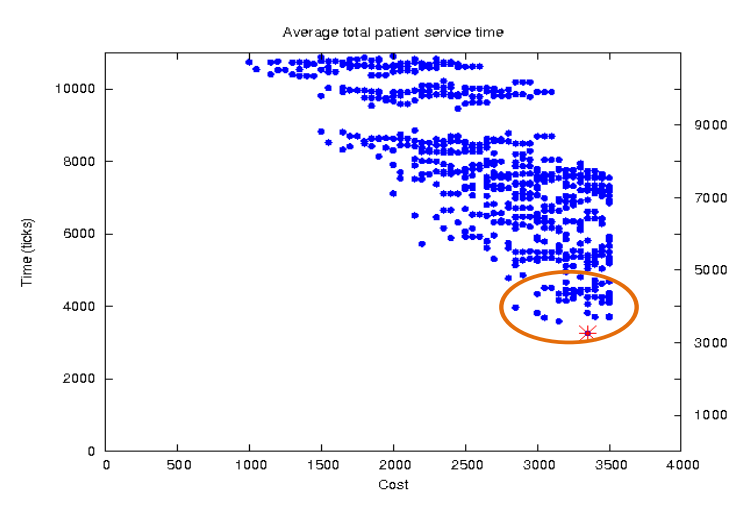

(b) Average minimum LoS with $\mathrm{P}=80 \%$.

Figure 3: The blue rounded points are the staff configurations that satisfy the constraint cost. Staff configurations that got the average minimum LoS are shown as green and red asterisks.

From Figure 3a, it can be seen that there are three different staff configurations which obtain the minimum LoS, but with different costs. Also, in the same Figure 3a, it can be seen that there are many other staff configurations that are quite close to the minimum LoS, but with less cost.

Table 2: Staff members with their associated salary and time according to their kind, and the maximum quantity of them.

\begin{tabular}{ccccccc}
\hline Min & $€$ & Time (ticks) & \# staff & D & N & A \\
\hline 1 & 3,200 & 428 & 5 & $2 \mathrm{~S}$ & $2 \mathrm{~S}$ & $1 \mathrm{~S}$ \\
\hline 2 & 2,900 & 428 & 5 & $2 \mathrm{~S}$ & $1 \mathrm{~S}$ & $2 \mathrm{~S}$ \\
\hline 3 & 2,850 & 428 & 5 & $2 \mathrm{~S}$ & $1 \mathrm{~S}$ & $1 \mathrm{~S}, 1 \mathrm{~J}$ \\
\hline
\end{tabular}

In the other cases, where the patient arrival increases, i.e., has higher probability (P), there are only a few staff configurations around the minimum, or clearly only one. Not only does the patient arrival increase, but also the minimum average patient LoS, as expected, as well as the cost of the staff configuration, and 
Table 3: Staff members with their associated salary and time according to their kind, and the maximum quantity of them.

\begin{tabular}{ccccccc}
\hline Min & $€$ & Time (ticks) & \# staff & D & N & A \\
\hline 1 & 3,350 & 3,266 & 7 & $1 \mathrm{~S}, 3 \mathrm{~J}$ & $2 \mathrm{~J}$ & $1 \mathrm{~J}$ \\
\hline
\end{tabular}

also the standard deviation of the average patient LoS. The amount of patients increases at waiting rooms, both WR1 and WR2 at times t1, t2, t3, and 4 (each time represents every 6,000 ticks of simulation), and finally the amount of unattended patients increases as well. In Table 4 all these results are shown. Comparing standard deviation results show us that the variability of LoS increases as the patient arrival $\mathrm{P}$ increases, but such variability is not linear. It is noticed that when the patient arrival $\mathrm{P}$ is high, $80 \%$, patients in waiting rooms WR1 and WR2 increase, i.e., the ED become overcrowded.

Table 4: Results for the best average minimum LoS for each of the four considered scenarios.

\begin{tabular}{lccccccc}
\hline $\mathbf{P}$ & $\mathbf{T 1}$ & $\sigma_{T 1}$ & $€$ & \# attended \# unattended & WR1 & WR2 \\
\cline { 5 - 8 } (ticks) & & & patients & patients & t1, t2, t3, t4 t1, t2, t3, t4 \\
\hline 20 & 428 & $48(11 \%)$ & 2850 & 83 & 1 & $0,0,0,0$ & $0,0,0,0$ \\
\hline 80 & 3,266 & $1,670.4(51.2 \%)$ & 3,350 & 294 & 100 & $8,19,32,43$ & $12,25,37,51$ \\
\hline
\end{tabular}

It is worth pointing out that each of the plotted points were obtained running the simulator as many times as there are points, i.e., for each case the optimum red and green points were obtained through the exhaustive search technique, which implies that all the scenarios that satisfy the restrictions should be executed on the simulator. This implies a lot of computing time, even though parametric executions, through NetLogo's BehaviorSpace tool were used. Therefore, when an entire and not simplified ED is used, the number of cases that have to be tested through exhaustive search will be very large. In order to reduce computational cost an intelligent search, other than exhaustive, must be used.

\section{PROPOSED REDUCED SCHEME}

Notice in Figure 3a and its corresponding Table 2 that there are different staff configurations that attained the average minimum LoS, but with different costs, as well as that there are many other staff scenarios that are quite close to the minimum time, but with less cost, i.e., these other solutions are sub-optimal. On the other hand, where the patient arrival increases, there are only a few staff configurations around the minimum, as can be seen in Figure $3 \mathrm{~b}$ and Table 3. Taking these results into account, i.e., there exist several configurations lying nearby the region where the optimum is; such configurations or scenarios are located inside the red ellipses in Figures $3 \mathrm{a}$ and $3 \mathrm{~b}$. The red ellipses delimits a region, which is not only where the optimum is, but also where sub-optima can be found, and some of such sub-optima could be selected instead of the optimum with a little less LoS, but with cheaper cost.

It was identified that ordering simulation points (ordered by ticks or weighted pipeline time ( $\mathbf{t}^{*}$, using Equation (2) and also shown in Figure 4 ) of staff configuration), before plotting it resembles the operation of a pipeline. The ordered and non-ordered data are shown in Table 5 (similar tables were obtained for nurses, and admission personnel). Thus, a computationally simplified model of ED as a pipeline scheme was implemented in order to verify this assumption. The pipeline scheme proposed is described as follows: patients continue arriving to the service, when only one admission personnel, nurse and doctor are available; therefore, the ED can be seen as a single queue system, whereas when more than one staff is available, patients are served as if they are in many parallel servers. When the pipeline is full, i.e., all sanitarian staff (admission personnel, nurses and doctors are busy ) and the previous queues of triage and diagnostic are occupied, then every certain ticks one patient leaves the ED (ideally, because it depends on patient's acuity).

MC is used in similar way, but in this case a specific staff configuration is selected to be tested and verified if it is the optimum; whereas the number of combination of EDs' staff is increased the standard error is reduced, i.e., it is closer to the optimum in the region nearby. 
Therefore, the proposal is to use a computationally simplified model of ED which consists of: a) pipelines, i.e., the ED can be viewed as a set of pipelines connected among them, and b) MC technique in order to identify the limited region where the optimum lies. This can be observed in Figure 4 . These alternative schemes to find the optimum, or the region where the optimum is, can lead us to find the optimum or sub-optimum values using an intelligent strategy, reducing the computing time and the region where the optimum lies. Once the region has been found using this scheme, the Agent-Based ED simulator previously described can be executed exhaustively on such a reduced region.

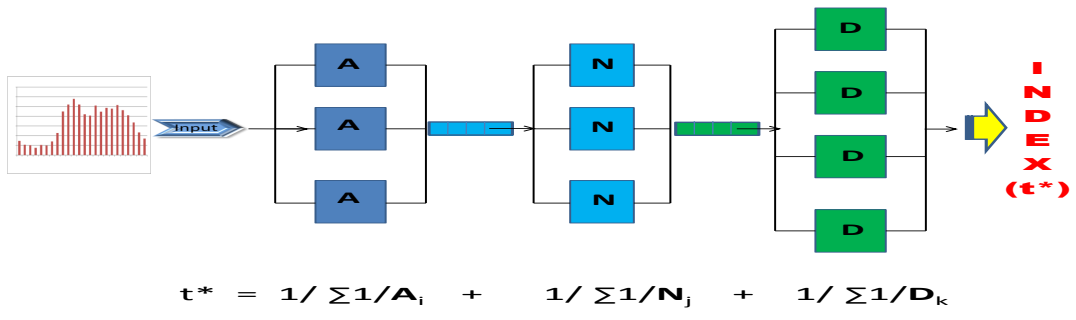

Figure 4: The proposal pipeline ED computationally simplified.

In Figure 5, a scattered 3D graph of the Figure 3a is shown. From this graph nothing can be concluded about a region where the optimum and sub-optimum are, while the same data but previously ordered according to the first column of the Table 5 and obtained through the computationally simplified proposed model of ED, Pipeline scheme, is shown in the Figures 6 and 7. In these graphs a region, where the optimum and sub-optima are, is clearly identified, specially in the latter, which isosurfaces are shown. In this region an exhaustive but reduced search is done, in order to find out the optimum using an intelligent strategy. Similar regions are found using MC scheme.

Table 5: Ordering data according to working time of each staff configuration of Doctors.

\begin{tabular}{ccccccccc}
\hline Ordered $\mathbf{t}^{*}$ & Non ordered & $\mathbf{D R}_{1}$ & $\mathbf{D R}_{2}$ & $\mathbf{D R}_{3}$ & $\mathbf{D R}_{4}$ & $€$ & $\mathbf{t}($ ticks) & $\mathbf{t}^{*}$ (ticks) \\
\hline 1 & 10 & DS & DS & DS & DS & 4,000 & 260 & 65 \\
\hline 2 & 14 & DS & DS & DS & DJ & 3,500 & 350 & 69.5 \\
\hline 3 & 13 & DS & DS & DJ & DJ & 3,000 & 350 & 74.6 \\
\hline 4 & 12 & DS & DJ & DJ & DJ & 2,500 & 350 & 80.5 \\
\hline 5 & 6 & DS & DS & DS & - & 3,000 & 260 & 86.7 \\
\hline 6 & 11 & DJ & DJ & DJ & DJ & 2,000 & 350 & 87.5 \\
\hline 7 & 9 & DS & DS & DJ & - & 2,500 & 350 & 94.8 \\
\hline 8 & 8 & DS & DJ & DJ & - & 2,000 & 350 & 104.6 \\
\hline 9 & 7 & DJ & DJ & DJ & - & 1,500 & 350 & 116.7 \\
\hline 10 & 3 & DS & DS & - & - & 2,000 & 260 & 130 \\
\hline 11 & 5 & DS & DJ & - & - & 1,500 & 350 & 149 \\
\hline 12 & 4 & DJ & DJ & - & - & 1,000 & 350 & 175 \\
\hline 13 & 1 & DS & - & - & - & 1,000 & 260 & 260 \\
\hline 14 & 2 & DJ & - & - & - & 500 & 350 & 350 \\
\hline
\end{tabular}

\section{CONCLUSIONS AND FUTURE WORK}

This paper presents a concrete example that uses a promising approach of an agent-based model to simulate Healthcare Emergency Departments (ED), whose complexity and dynamic nature make them difficult to characterize. The model uses Moore state machines based agents which act and communicate within a defined layout. In order to verify and validate the model an initial simulation has been made. An index defined as the minimum patient length of stay $(\operatorname{LoS})$ was set to evaluate the operation of the agent-based Emergency Department simulator. The search of the optimum LoS was done through an exhaustive search technique, which implies a large searching time. The obtained results are encouraging, since they not 


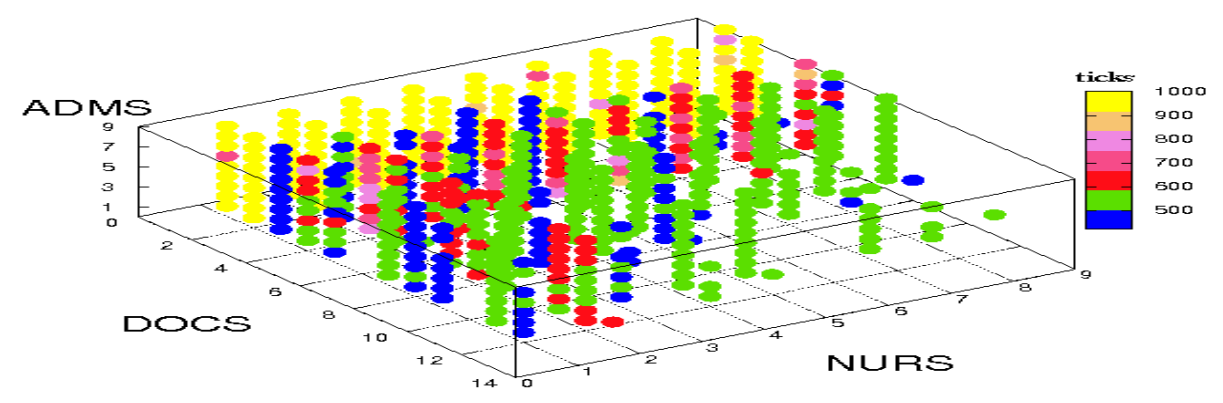

Figure 5: Scattered 3D graph using the second column of the Table 5 where there is a lack of order of staff configuration. The axes are labeled by the number of combinations of each ED staff: up to 9 for Nurses and Admission personnel and up to 14 for Doctors.

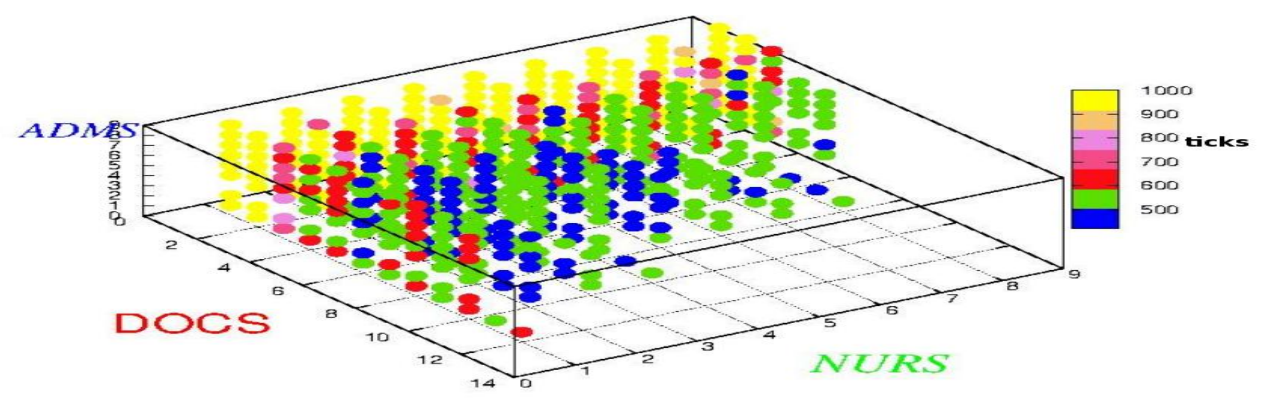

Figure 6: Scattered 3D graph obtained through the proposed reduce ordered scheme Pipeline. The axes are labeled by the number of combinations of each ED staff: up to 9 for Nurses and Admission personnel and up to 14 for Doctors.

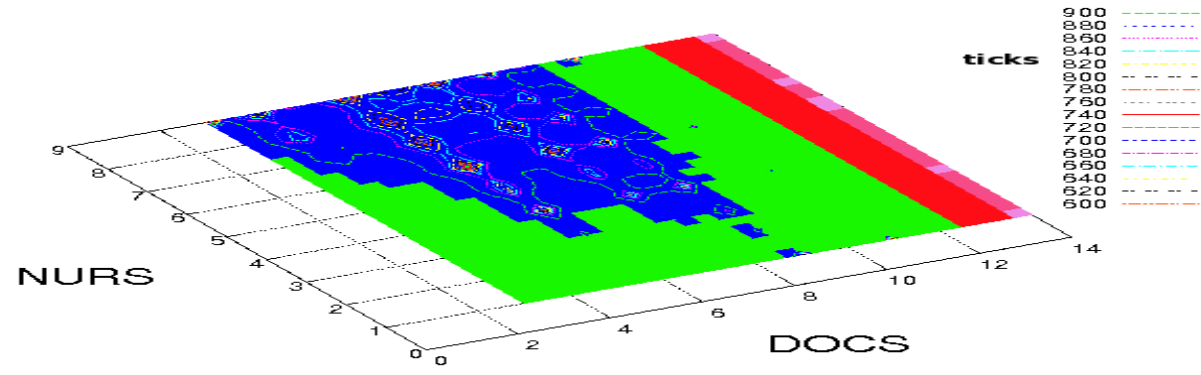

Figure 7: Isosurfaces graph obtained through the proposed ordered reduce scheme Pipeline. The axes are labeled by the number of combinations of each ED staff: up to 9 for Nurses and Admission personnel and up to 14 for Doctors.

only showed that as expected, the larger number and experienced ED staff, the less average patient LoS is estimated, but also showed interesting results when its standard deviation is analyzed. Simulation allows to understand and analyze better the problem. However, even with the pretty small problem size studied the number of combinations are large, as well as the execution time. Moreover, the computational resources that this problem will demand in order to perform statistical sensitivity analysis are huge. Therefore, a better optimization technique or approach, rather than exhaustive search technique, must be used. This 
approach is using a computationally simplified model of the ED as MC and Pipeline schemes in order to identify the region where the optimum is, and do a reduced exhaustive search, in this limited region. The results using such approaches are not only promising, but also computationally not as intense as the computing using exhaustive search technique.

As future work, the proposed alternatives methodologies MC and Pipeline schemes have to be tested with a more detailed ED facility, as well as more sanitarian staff and more realistic budgets. Moreover, due to the multi-dimensional nature of the problem, i.e., large amount of individuals as well as the number of states in the state machine of each individual, different time periods, and the multi- objective optimization problem a large amount of parameter values should be computed; therefore, the use of high performance computing will be mandatory.

\section{ACKNOWLEDGMENTS}

This research has been supported by the MICINN Spain, under contract TIN2007-64974 and the MINECO (MICINN) Spain, under contract TIN2011-24384.

\section{REFERENCES}

Ahmed, M. A., and T. M. Alkhamis. 2009. "Simulation optimization for an emergency department healthcare unit in Kuwait". European Journal of Operational Research 198 (3): 936-942.

Fu, M. C. 2002. "Feature Article: Optimization for simulation: Theory vs. Practice". INFORMS Journal on Computing 14 (3): 192-215.

Hancock, W. M., and P. F. Walter. 1979. "The use of computer simulation to develop hospital systems". SIGSIM Simul. Dig. 10 (4): 28-32.

Hutzschenreuter, A. K., P. A. Bosman, and H. Poutré. 2009. "Evolutionary Multiobjective Optimization for Dynamic Hospital Resource Management". In Evolutionary Multi-Criterion Optimization, edited by M. Ehrgott, C. Fonseca, X. Gandibleux, J.-K. Hao, and M. Sevaux, Lecture Notes in Computer Science, 320-334. Springer Berlin / Heidelberg.

Hutzschenreuter, A. K., P. A. N. Bosman, I. Blonk-Altena, J. van Aarle, and H. La Poutré. 2008. "Agent-based patient admission scheduling in hospitals". In Proceedings of the 7th international joint conference on Autonomous agents and multiagent systems: industrial track, AAMAS '08, 45-52. Richland, SC: International Foundation for Autonomous Agents and Multiagent Systems.

Institute of Medicine (IoM), and Committee on the Future of Emergency Care in the United States Health System. 2007. Hospital-Based Emergency Care: At the Breaking Point. Washington, D.C.: The National Academies Press.

Jones, S. S., and R. S. Evans. 2008. "An Agent Based Simulation Tool for Scheduling Emergency Department Physicians". In AMIA Annual Symposium proceedings, AMIA Symposium, 338-342.

Jun, J. B., S. H. Jacobson, and J. R. Swisher. 1999, February. "Application of discrete-event simulation in health care clinics: A survey". Journal of the Operational Research Society:109-123.

Laskowski, M., and S. Mukhi. 2008. "Agent-Based Simulation of Emergency Departments with Patient Diversion". In Electronic Healthcare, edited by D. Weerasinghe, O. Akan, P. Bellavista, J. Cao, F. Dressler, D. Ferrari, M. Gerla, H. Kobayashi, S. Palazzo, S. Sahni, X. S. Shen, M. Stan, J. Xiaohua, A. Zomaya, and G. Coulson, Lecture Notes of the Institute for Computer Sciences, Social Informatics and Telecommunications Engineering, 25-37: Springer Berlin Heidelberg.

Lynn, S. G., and A. L. Kellermann. 1991. "Critical decision making: Managing the emergency department in an overcrowded hospital". Annals of Emergency Medicine 20 (3): 287-292.

Persson, J. A., P. Davidsson, S. J. Johansson, and F. Wernstedt. 2005. "Combining Agent-Based Approaches and Classical Optimization Techniques". In EUMAS, edited by M. P. Gleizes, G. A. Kaminka, A. Nowé, S. Ossowski, K. Tuyls, and K. Verbeeck, 260-269: Koninklijke Vlaamse Academie van Belie voor Wetenschappen en Kunsten. 
Ruohonen, T., P. Neittaanmaki, and J. Teittinen. 2006, December. "Simulation Model for Improving the Operation of the Emergency Department of Special Health Care". In Proceedings of the 2006 Winter Simulation Conference, edited by L. F. Perrone, F. P. Wieland, J. Liu, B. G. Lawson, D. M. Nicol, and R. M. Fujimoto, 453-458. Piscataway, New Jersey: Institute of Electrical and Electronics Engineers, Inc.

Saunders, C. E., P. K. Makens, and L. J. Leblanc. 1989. "Modeling emergency department operations using advanced computer simulation systems". Annals of Emergency Medicine 18 (2): 134-140.

Stainsby, H., M. Taboada, and E. Luque. 2009. "Towards an Agent-Based Simulation of Hospital Emergency Departments". Services Computing, IEEE International Conference on 0:536-539.

Taboada, M., E. Cabrera, M. L. Iglesias, F. Epelde, and E. Luque. 2011. "An Agent-Based Decision Support System for Hospitals Emergency Departments". Procedia CS 4:1870-1879.

Wang, L. 2009, April. "An agent-based simulation for workflow in Emergency Department". In Systems and Information Engineering Design Symposium, 2009. SIEDS '09., 19-23.

Weng, S.-J., B.-C. Cheng, S. T. Kwong, L.-M. Wang, and C.-Y. Chang. 2011, December. "Simulation Optimization for emergency department resources allocation". In Proceedings of the 2011 Winter Simulation Conference, edited by S. Jain, R. R. Creasey, J. Himmelspach, K. P. White, and M. Fu, 1231-1238. Piscataway, New Jersey: Institute of Electrical and Electronics Engineers, Inc.

U. Wilensky 1999. "Netlogo". Northwestern University, Center for Connected Learning and Computer-Based Modeling.

\section{AUTHOR BIOGRAPHIES}

EDUARDO CABRERA is a PhD student of High Performance Computing at Universitat Autònoma de Barcelona (UAB). He received his master degree from the Computer Architecture and Operating Systems (CAOS) at UAB and a B.S. degree in Computer Engineering from Universidad Nacional Autónoma de México (UNAM). His research interests include modeling and simulation, agent-based models, parallel computer architectures, parallel and distributed simulation, and optimization. His email address is ecabrera@caos.uab.es.

MANEL TABOADA is a full professor in the Tomàs Cerdà Computer Science School ( UAB ), Spain. He studied Business Administration at the University of Barcelona, Spain. From 2009 is Ph.D. student at CAOS Department of the UAB. He is mainly interested in the simulation-based decision support for the operational management of Health care units and organizations, and specially the Hospital Emergency Departments. His email address is manel.taboada@eug.es.

EMILIO LUQUE is a full professor in the Computer Architecture and Operating System (CAOS) Department at UAB, Spain. From 1994 he has been involved, leading his group, in several research projects founded by the European Union (EU), the Spanish government and different industries. His major research areas are: parallel computer architecture, parallel and distributed simulation, performance prediction and efficient management of multi-cluster-multi-core systems and fault tolerance in parallel computers and has published more than 40 papers in technical journals and more than 180 papers at international conferences.

FRANCISCO EPELDE, MD, Ph D. Internal Medicine Doctor. Currently Medical Coordinator of the Short Stay Unit in the Emergency Department in the Hospital of Sabadell. Consorci Hospitalari i Universitari Parc Taulí (Taulí) in Sabadell (Spain) and professor of Emergency Medicine in the Medicine School of the UAB. Hi email address is fepelde@tauli.cat.

M. LUISA IGLESIAS MD. Currently Director of the Emergency Department in the Hospital de Sabadell, Tauli and professor of clinical toxicology in the Medicine School of the UAB. Her email address is miglesias@tauli.cat. 